\title{
Frontières
}

\section{Nouvelles parutions}

\section{Ngoc-Thanh Phan Nguyen}

Volume 13, numéro 2, printemps 2001

URI : https://id.erudit.org/iderudit/1074469ar

DOI : https://doi.org/10.7202/1074469ar

Aller au sommaire du numéro

Éditeur(s)

Université du Québec à Montréal

ISSN

1180-3479 (imprimé)

1916-0976 (numérique)

Découvrir la revue

Citer ce document

Phan Nguyen, N.-T. (2001). Nouvelles parutions. Frontières, 13(2), 94-95. https://doi.org/10.7202/1074469ar

Ce document est protégé par la loi sur le droit d'auteur. L'utilisation des services d'Érudit (y compris la reproduction) est assujettie à sa politique d'utilisation que vous pouvez consulter en ligne.

https://apropos.erudit.org/fr/usagers/politique-dutilisation/
Cet article est diffusé et préservé par Érudit.

Érudit est un consortium interuniversitaire sans but lucratif composé de l’Université de Montréal, l'Université Laval et l'Université du Québec à Montréal. Il a pour mission la promotion et la valorisation de la recherche. https://www.erudit.org/fr/ 
BERMAN, Michael R., Parenthood lost: healing the pain after miscarriage, stillbirth, and infant death, Westport, Conn., Bergin \& Garvey, 2001, 247 pages.

BROOKE, Jill, Don't let death ruin your life: a practical guide to reclaiming happiness after the death of a loved one, New York, E. P. Dutton, 2001, 270 pages.

CHARPENTIER, Gérard, Les malades et leurs émotions, Mortefontaineen-Thelle (Oise), Mortaigne, 2000, 304 pages.

CHOQUE, Stella et CHOQUE, Jacques, Animations pour les personnes âgées: 400 exercices pratiques et Iudiques, Rueil-Malmaison (Hautsde-Seine), Lamarre, coll. « Pratiquer », 2000, VI-169 pages.

Comprendre le traumatisme: une approche psychanalytique, LamorPlage (Morbihan), Éd. du Hublot, coll. "Tavissork Clinic », 2001, 222 pages.

\section{NOUVELLES} PARUTIONS

Ngoc-Thanh Phan-Nguyen

AIKEN, Lewis R., Dying, Death, and Bereavement, 4th ed., Mahwah, N.J., L. Erlbaum, 2001, 398 pages.

ASSOCIATION DE GESTION DES MOYENS DU CODERPA 44 (LoireAtlantique), Accueil et soins des personnes âgées: les structures de Loire-Atlantique : 2000-2001, Nantes, Agm-Coderpa, 2000, 148 pages.

AUGER, Jeannette A., Social Perspectives on Death and Dying, Halifax, N.-É., Fernwood, 2000, 240 pages.

BARRY, Aboubacar, Le corps, la mort et l'esprit du lignage: l'ancêtre et le sorcier en clinique africaine, Paris, L'Harmattan, coll. "Santé, sociétés et culture », 2000, 270 pages.
DEVIES, Penelope J. E, Death and the Emperor: Roman Funerary Monuments, from Augustus to Marcus Aurelius, New York, Cambridge University Press, 2000, 288 pages.

DELL'ANNELLO, Marie etDESLAURIERS, Gilles, Rencontre entre un thérapeute et une famille en deuil: la mort d'Yves, Paris, L'Harmattan, coll. "Au delà du témoignage », 2000, 178 pages.

DIRICQ, Catherine et PAYEN, MarieChristine, L'Euthanasie : à partir de quatre histoires vécues, Bruxelles, Labor, coll. "La Noria », 120 pages.

DUVAL, Roselyne et MOINGEON, Guillaume, Vivre ou survivre, Lanrodec (Côte-d'Armor), Scripta, 2001, 147 pages.

FERGANE, Bernard et JEANMOUGIN, Chantal, Douleur: soins préventifs et prise en charge, Paris, Flammarion médecine-sciences, 2000, VIII-111 pages.

Association des diplômés
en études sur la mort (ADEM) $\begin{aligned} & \begin{array}{l}\text { ADEM, Bureau des diplômés, local B-1105 } \\ \text { Université du Québec à Montréal } \\ \text { Case postale 8888, succursale Centre-ville } \\ \text { Montréal (Québec) H3C 3P8 }\end{array} \\ & \begin{array}{l}\text { Adhésion, incluant 2 numéros de la revue Frontières } \\ \square \text { Canada } 40 \$\end{array} \\ & \begin{array}{l}\text { Prénom et nom: } \\ \text { Adresse: }\end{array} \\ & \begin{array}{l}\text { Code postal: } \\ \text { Tél. rés.: }()\end{array} \\ & \begin{array}{l}\text { Profession: } \\ \hline\end{array}\end{aligned}$


FORTIN, Bruno, Côtoyer la souffrance des personnes âgées, Saint-Laurent (Québec) Fides, 2000, 120 pages.

GAGNON, Éric et SAILLANT, Francine, De la dépendance et de l'accompagnement: soins à domicile et liens socia ux, Paris, L'Harmattan; Québec, Presses de I'Université Laval, coll. "Sociétés, cultures et santé ", 2000, 232 pages.

HOLSTENSSON, Lucette et RIOUFOL, Marie-Odile, Besoins affectifs et sexualité des personnes âgées en institution: le savoir et le "comment faire " face à un tabou, Paris, Masson, coll. "Formation et pratique de l'aide-soignant », 2000, V-97 pages.

IRELAND, Marie, Apprivoiser le deuil: surmonter la perte d'un être cher, Paris, Presses du Châtelet, 2001, 216 pages.

LESCHEMELLE, Pierre, Les années de trop pour mourir dignement, Paris, Imageo, 2000, 200 pages.

LIFTON, Robert Jay et MITCHELL, Greg, Who owns death?: Capital punishment, the American conscience, and the end of executions, New York, W. Morrow, 2000, 270 pages.

LÔO, Henri et LÔO, Pierre, La dépression, $4^{\mathrm{e}}$ éd. mise à jour, Paris, Presses universitaires de France, coll. "Que sais-je ? », 2000, 127 pages.

KUBLER-ROSS, Elisabeth et KESSLER, David, Life lessons: Two experts on death and dying teach us about the mysteries of life and living, New York, C. Scribner, 2000, 224 p.

MC KENZIE, Kwame, Dépression, Alleur (Belgique), Marabout, coll. "Guides de médecine familiale", 2001, 80 pages.

MANOUKIAN, Alexandre, Lessoignants et les personnes âgées, $2^{\mathrm{e}}$ éd., RueilMalmaison (Hauts-de-Seine), Lamarre, coll. « Pratiques », 168 pages.

MANOUKIAN, Alexandre et MASSEBEUF, Anne, La relation soignantsoigné, $2^{\mathrm{e}}$ éd., Rueil-Malmaison (Hauts-de-Seine), Lamarre, coll. "Pratiques", 158 pages.

MARCILLAt, Hervé, Vieillesse et société: le rendez-vous manqué: la dépendance des personnes âgées en question, Toulouse Erès, coll. « Pratiques du champ social », 2000, 158 pages.

MARTIN, Claude (dir. de publication), La dépendance des personnes âgées, Renne, Presses universitaires de Rennes, coll. « Res Publica », 2001, 280 pages.

NOEL, Brook et BLAIR, Pamela D., I wasn't ready to say goodbye: Surviving, coping \& healing after the sudden death of a loved one, Beverley Hills, Calif., Champion Press, 2000, 285 pages.
TAVERNIER, Monique, Les soins palliatifs , $4^{\mathrm{e}}$ éd. mise à jour, Paris, Presses universitaires de France, coll. "Que sais-je ? ", 2000, 128 pages.

NEROME, Simone, DOLOMENI, Pierre et BOUISSON, Valérie, Hygiène et soins infirmiers aux personnes atteintes de maladies infectieuses et aux personnes atteintes par le VIH, Paris, Ellipses-Marketing, coll. "IFSI, Réussir le diplôme d'État », 2000, 300 pages.

RAVEL, Claire de, Les services aux personnes âgées, Vanves, Entrepreneuriat, coll. « Opticréa; Découvrir le marché ", 2000, 66 pages.

ROSENBLATT, Paul C., Help your marriage survive the death of a child, Philadelphia, Temple University Press, 2000, 184 pages.

SAMAOLI, Omar et al. Vieillesse, démence et immigration: pour une prise en charge adaptée des personnes âgées migrantes en France, au Danemark et au Royaume-Uni, Paris, L'Harmattan, coll. " Minorités et société », 2000, 256 pages.

SECRETAN, Jacques, Condamné à mort au Texas: témoignages du couloir de la mort, Paris, L'Harmattan, 200, 205 pages.

SCHENK, Ronald, Dark light: The appearance of death in everyday life, Albany, N.Y., State University of New York Press, coll. "SUNY series, alternatives in psychology ", 2000, 180 pages.

SMITH, Wesley, J., Culture of death: The assault on medical ethics in America, San Francisco, Encounter books, 2000, 250 pages.

THIÉBAUT, Danielle, Les personnes âgées et la désorientation: comprendre, aider, prévenir, travailler en équipe, Paris, ESF éditeur, coll. "Actions sociales; Société », 2000, 107 pages.

YORK, Sarah, Remembering well: Rituals for celebrating life and mourning death, San Francisco, Calif., Jossey-Bass, 2000, 240 pages. 